\title{
Editorial
}

\section{Good Clinical Laboratory Practice (GCLP): Quality Demanding from Clinical Laboratories}

Good Clinical Practice (GCP) is an international ethical and scientific quality standard for designing, conducting, recording and reporting trials that involve the participation of human subjects. Compliance with this standard provides public assurance that the rights, safety and well-being of trial subjects are protected, consistent with the principles that have their origin in the Declaration of Helsinki (ICH GCP Guideline).

Good Laboratory Practice (GLP) is intended to promote the quality and validity of test data. It is a managerial concept covering the organizational process and the conditions under which laboratory studies are planned, performed, monitored, recorded and reported (OECD GLP Guideline).

Good Clinical Laboratory Practice (GCLP) applies those principles established under GLP for data generation used in regulatory submissions relevant to the analysis of samples from a clinical trial. At the same time it, ensures that the objectives of the GCP principles are carried out. This ensures the reliability and integrity of data generated by analytical laboratories. It is recognized that a number of countries are already applying the GCLP principles to the analysis of clinical trial samples. Indeed, it is possible within some of these countries for clinical laboratories to be accredited by the National Monitoring Authority.

As a developing country, Bangladesh is making advances in the field of technology, research, infrastructure and skilled manpower. However, much of these advances are limited to metropolitan cities and urban areas. As the use of laboratory tests (often expensive) are increasingly becoming a part of medical diagnosis and research, generation of quality data would be a cost-effective and ethically sound strategy.

For some years, it has been internationally recognized that clinical laboratories processing specimens from clinical trials require an appropriate set of standards to guide good practices. $^{1}$

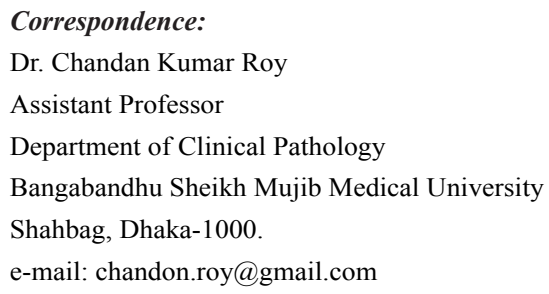

Some organizations and indeed, countries operate proficiency testing schemes to which laboratories subscribe. While these ensure the integrity of the analytical process, they may not assure compliance with GCP. This document is intended to provide a unified framework for sample analysis to lend credibility to the data generated and facilitate the acceptance of clinical data by regulatory authorities from around the world. It is important to recognize that the framework outlined in this document will be applied across a diverse set of disciplines involved in the analysis of samples from clinical trials. It is, therefore, important to understand that this framework should be interpreted and applied to the work of those organizations that undertake such analyses with the objective of assuring the quality of every aspect of the work that they perform. ${ }^{2}$

In April 2006, the Special Programme for Research and Training in Tropical Diseases TDR, sponsored by UNDP, UNICEF, the World Bank and WHO, convened a meeting of organizations engaged in clinical trials in disease endemic countries to discuss the applicability of these guidelines to their work. Invited organizations included Epicentre, Drugs for Neglected Diseases initiative (DNDi), the Foundation for Innovative New Diagnostics (FIND), and the Kenya Medical Research Institute (KEMRI). It was agreed that GCLP would be a valuable tool for improving and assuring quality laboratory practice in clinical trials in the tropical settings in which they work. It was recognized that the GCLP Guidelines were not widely available, and it was recommended that WHO/TDR publish the guidelines on its website as the standard for laboratories undertaking samples from TDRsupported clinical trials. The TDR Diagnostics Evaluation Expert Panel (DEEP) has since recommended GCLP as the standard for clinical laboratories involved in the evaluation of diagnostics for infectious diseases. ${ }^{3}$

Good Clinical Laboratory Practices (GCLP) outline the principles and procedures to be followed by medical laboratories involved in patient care and/or clinical research so as to provide consistent, reproducible, auditable, and reliable laboratory results which contribute to good patient care and promote a positive attitude toward testing from a patient's perspective. Laboratory tests are used to support diagnosis in patient care as well as medical research. The test results, therefore, should be reliable, accurate and reproducible. Generation of such 'quality' results involves a 
step wise process of meticulous planning, perfect execution and thorough checking of results by the whole team involved. International guidelines lay down the principles of Good Clinical Laboratory Practices (GCLP) to be followed by medical researchers to generate 'quality data' but do not address the issues of reporting 'quality' test results for day-today patient care.

Laboratory services are an integral part of disease diagnosis, treatment, monitoring response to treatment, disease surveillance programmes and clinical research. The World Development Report, 1993, regarded provision of Essential Health Technology as an important ingredient of Essential Clinical Services. Use of diagnostic techniques aid early diagnosis, enabling appropriate and prompt intervention, thereby reducing overall disease burden and promoting health. All laboratories are not equipped with facilities for carrying out complex investigations. The structure and function of a clinical laboratory varies according to the level of health care facility. Peripheral laboratories carry out simple tests, whereas higher centers are equipped with sophisticated technology and trained manpower to carry out complex investigations. Establishing a network between peripheral and higher laboratories allows collection of specimen at periphery and their storage and transport for testing at higher centers and communicating report to the peripheral center efficiently without actually having to transfer the patient. In the event of patient transfer, the higher centers do not need to repeat investigations carried out at the peripheral health center, thereby saving crucial time as well as cost and providing continuity in patient care. Networking between laboratories is also essential in disease surveillance programmes and outbreak investigations in order to obtain quick and reliable results.

In Bangladesh, the National Accreditation Board for Testing and Calibration Laboratories is going to provide accreditation services to medical laboratories by short time.

In biomedical research too, achieving a set standard of quality produces credible results and allows comparison between studies carried out at different institutes nationally and internationally. This saves enormous time, money and resources and prevents duplication of research work. The International Conference on Harmonization ( $\mathrm{ICH}$ ) provided 'Good Clinical Practices (GCP) Guidelines' which describe standards to be followed by researchers while designing, conducting and reporting trials involving human participants.

Good Clinical Laboratory Practices should be used by all laboratories where tests are done on biological specimens for diagnosis, patient care, disease control and research such as:

- Microbiology \& Serology

- Hematology \& Blood Banking
- Molecular Biology and Molecular Pathology

- Clinical Pathology

- Clinical Biochemistry

-Immunology (Immunohematology and

Immunobiochemistry)

- Histopathology/Pathology and Cytology

Infrastructure of laboratories should be planned according to the services provided by the laboratory. Appropriately trained and well organized laboratory staffs are key to the successful operation of a research facility. Systems are required to drive organizational structure, training and ongoing competency assessment to ensure appropriate accountability and communication during study conduction. ${ }^{4}$ Each laboratory should designate a Head of the laboratory, who should be overall in-charge of the daily functioning of the laboratory, including administration. A Quality Manager should be designated for monitoring and maintaining of day-to-day quality management system. Annual evaluations for the employee's overall performance of job responsibilities, duties, and tasks as outlined in the job description must be given to all laboratory personnel. ${ }^{5}$

Each laboratory should prepare an exhaustive list of equipment and consumables required and available for general functioning of the laboratory and specialized equipment for special tests. All equipment should be in good working condition at all time. Periodic inspection, cleaning, maintenance of equipment should be done. An equipment log book should be maintained for all major equipments. Laboratories should maintain necessary instructions for operation and maintenance of equipment in the form of Standard Operating Procedures (SOPs). A copy of SOP should be readily available. ${ }^{6,7}$

The SOP is a document, which contains detailed, written instructions describing the stepwise process and technique of performing a test or procedure in the laboratory. The SOP helps to ensure uniformity, consistency and control over the processes carried out. It ensures that the procedures are done in exactly the same way each time irrespective of the operator. The SOP should contain information on who can perform the test, their qualification and training, how to carry out the test including pre-analytical, analytical and postanalytical stages of test/procedure, laboratory conditions required for the test/ procedure, routine care and maintenance of equipment, precautions and safety instructions, trouble shooting measures, waste disposal and linkage with reference laboratories. The SOP should be simple and written in an easy to understand language. ${ }^{8}$

The reagents, chemicals and consumables should be stored under appropriate environmental conditions. Quality of newly purchased reagents should be validated against suitable control/reference material prior to use. Validation data should 
be properly documented.

In-house prepared reagents should also be checked periodically for stability and a record of the same should be maintained. Microbiology laboratories should check activity/potency of each lot of antibiotic sensitivity discs before using and at least weekly thereafter with reference strains. Other microbiological consumables such as strips etc. used for identification should be checked against reference strains. Laboratories testing microbiology specimens should check the quality of media by using appropriate reference strain and $\mathrm{pH}$ of the media. ${ }^{9}$

Specimen collection is the first phase of interaction between the patient and the laboratory. Appropriate counselling should be done before specimen collection and consent taken whenever needed. Attention should be paid to patient's sensibilities during the entire process. Any error in specimen collection can lead to erroneous results. It is, therefore, considered an important step of good clinical laboratory practice and is referred to as "preanalytic control". Laboratory should have a "primary specimen collection manual", containing information on patient preparation before specimen collection (if any), exact methodology of specimen collection, labelling, handling, transportation and storage of the specimens. In addition, the laboratory should provide adequate and appropriate information/instructions to patients wherever necessary. All preanalytical factors that may influence the test results should be identified. The manual should include guidelines on specimen collection including preservation for histopathological examination. These manuals should be available for reference and should be used for training of staffs engaged in specimen collection. ${ }^{9}$

The requisition form should be completed by the doctor requesting the tests and sent along with the specimen/patient to the laboratory. It should contain the patient's identity, age, location, date of specimen collection and the investigations requested. The referring doctor should be encouraged to mention the provisional or working diagnosis and relevant clinical and treatment history in the space provided. Worksheet is essentially a form provided to the analyst along with the specimen. ${ }^{10}$

Results should be reported clearly, without any errors, specifying measurement procedure, where appropriate and units of measurement as recommended by professional societies such as International Council for Standardization in Haematology, International Society of Haematology \& International Federation of Clinical Chemistry and Laboratory Medicine. Laboratories should maintain a record of specimens which were rejected prior to analysis. Rejection statistics (eg. number of hemolyzed specimen etc.) along with reason for rejection and person responsible for rejection should be maintained. ${ }^{10}$

Laboratory data management includes recording details of the patient, findings of analysis, reporting of results and archiving the data for future reference. Recording data allows smooth functioning of the internal quality control measures, internal audit and external quality assessment. From the point of view of management, absence of record implies that the work was never done. ${ }^{10}$

Personnel working in laboratories may be exposed to risks from various chemicals, infectious materials, fire hazard, gas leak etc. The environment is also at risk of being contaminated by hazardous materials used and wastes generated in the laboratory. Safety in laboratories, therefore, includes protection of the staff and the environment from hazardous materials. ${ }^{11,12}$

Personnel working in clinical and/or research laboratories or engaged in biomedical sciences or research should be aware of their ethical responsibilities and comply with the ethical code of conduct.

Quality Assurance (QA) is the total process whereby the quality of laboratory reports can be guaranteed. Incorrect Laboratory results may be due to errors occuring during specimen collection (pre-analytical stage), testing (analytical stage) and/or while reporting and interpreting (post-analytical stage) test results. Whereas, Internal Quality Control (IQC) refers to the process of minimizing analytical errors, QA encompasses procedures adopted for minimizing errors that may occur at any stage provide QA, all laboratories must have a Quality Assurance Programme (QAP) in place. ${ }^{13,14,}$ 15,16

Internal Quality Control (IQC) includes the following: - Recognition of errors which arise within the laboratory during analytical stage (testing).

$\varnothing$ Taking steps to minimize errors.

$\varnothing$ Equipment \& method calibration, method validation

- Laboratories should perform IQC

$\varnothing$ Every day on tests run daily

$\varnothing$ Every time the tests are run in case of infrequently used tests.

- Quality control checks should be employed for both quantitative and qualitative tests.

External Quality Assessment (EQA) refers to a system in which the performance of a laboratory is assessed periodically and retrospectively by an independent external agency to indicate to the laboratory staff of any shortcomings in performance. It indicates a need for improving and/or changing IQC procedures. An organizing agency or laboratory sends identical specimens to all participating laboratories for 
testing by methods routinely adopted by them. The results from the participating laboratories are received by the organizing agency and compared with a "correct" answer retrospectively and a performance score is assigned. All the participating laboratories are identified by a code and reports issued to the participants contain the performance score of all participating laboratories including its own score. This allows a comparison of quality between laboratories and thus describes the "state of the art" for that area of laboratory work encompassed by EQA programmes. ${ }^{14,} 15$

Audit is a process of critical review of the functioning and evaluation of services. Internal audit is the systematic, independent and documented process for obtaining audit evidence and evaluating it objectively to determine the extent to which the specific criteria are complied with. Internal audit can be effectively carried out by examining documents, specimens, equipment, environmental conditions, examination procedures and personnel competence. Effective internal audit will identify the problems and weak points in the system and suggest remedial measures.

The GCLP standards were developed to bring together multiple guidance and regulatory information, as they apply to clinical research and to fill a void of a single GCLP reference for global clinical research laboratories, with regard to laboratories that support clinical trials such as those that perform protocol-mandated safety assays, process blood, and perform immune monitoring assays for candidates on a product licensure pathway. To maintain a GCLP environment for a clinical trial it is critical that all of the key GCLP elements are in place and operational. These elements include organization and personnel, testing facilities, appropriately validated assays, relevant positive and negative controls for the assays, a system for recording, reporting and archiving data, a safety program tailored to personnel working in the laboratory, an information management system that encompasses specimen receipt/ acceptance, storage, retrieval and shipping and an overall quality management plan. The most appropriate way to ensure compliance with GCLP guidance is to audit laboratories. Because key decisions regarding the advancement of products are based on laboratory-generated data obtained from specimens collected during the trials, GCLP compliance is critical. Such compliance will assist laboratories in ensuring accurate, precise and reproducible data are produced that guarantee sponsor confidence, and stand under regulatory agency review.

Adoption of GCLP guidelines by laboratories of public sector, private sector and research institutions will be a step forward in the betterment of health care services and health research.
A comprehensive version of the GCLP standards with accompanying templates and examples is available at [http://www3.niaid.nih.gov/research/resources/DAIDSClinRs $\mathrm{rch} / \mathrm{PDF} / \mathrm{labs} / \mathrm{GCLP.pdf}]$

\section{Dr. Chandan Kumar Roy}

Assistant Professor

Department of Clinical Pathology

Bangabandhu Sheikh Mujib Medical University

Shahbag, Dhaka-1000.

\section{References:}

1. Stevens W. Good Clinical Laboratory Practice (GCLP): The need for a hybrid of Good Laboratory Practice and Good Clinical Practice guidelines/standards for medical testing laboratories conducting clinical trials in developing countries. Quality Assurance, 10: 83-89, 2003.

2. Stiles, T. Grant, V. Mawby, N. BARQA. Good Clinical Laboratory Practice (GCLP): A Quality System for Laboratories that undertake the Analyses of Samples from Clinical Trials. 2003. Webpage at: http:// www.barqa.com (accessed on December 2010)

3. Banoo S, Bell D, Bossuyt P, Herring A, Mabey D, Poole F: (The TDR Diagnostics Evaluation Expert Panel). Evaluation of diagnostic tests for infectious diseases: general principles. Nature Reviews in Microbiology, September 2006 (S21). Webpage at :http://www.nature.com nrmicro/journal/v6/n11 supp/abs/nrmicro 1523.html (accessed on December 2010)

4. College of American Pathologists. Laboratory General Checklist. College of American Pathologists; Oct 2006. Webpage at : http: //www.cap.org/apps/docs/ laboratory_accreditation/checklists/ laboratory_general_october2006.pdf (accessed on December 2010)

5. 42 CFR. Part 493, Subpart M (493.1413), Standard; Technical consultant responsibilities. Oct. 2005

6. 42 CFR. Part 493, Subpart K (493.1254), Standard: Maintenance and function checks. Oct. 2005

7. College of American Pathologists. Chemistry and Toxicology Checklist. College of American Pathologists; Oct. 2006 CHM.23300

8. 42 CFR. Part 493, Subpart K (493.1251), Standard: Procedure manual. Oct. 2005

9. 42 CFR. Part 493, Subpart K (493.1256), Standard: Control procedures. Oct. 2005 
10. 42 CFR. Part 493, Subpart J (493.1105), Standard: Retention requirements. Oct. 2005

11. 49 CFR. Part 173, Subpart E (173.199), Category B infectious substances. Oct. 2006 http:// www.access.gpo.gov/nara/cfr/waisidx_06/49cfr173_06.html

12. 29 CFR. Part 1910, Subpart Z (1910.1030), Bloodborne pathogens. Jul. 2005 http:// www.access.gpo.gov/nara/cfr/waisidx_05/29cfr1910a_05.html

13. 42 CFR. Part 493, Subpart K (493.1289), Standard: Analytic systems quality assessment. Oct. 2005

14. 42 CFR. Part 493, Subpart K (493.1299), Standard: Postanalytic systems quality assessment. Oct. 2005

15. 42 CFR. Part 493, Subpart K (493.1239), Standard: General laboratory systems quality assessment. Oct. 2005

1642 CFR. Part 493, Subpart K (493.1249), Standard: Preanalytic systems quality assessment. Oct. 2005 